\title{
Implicit Schur Complement for Model Order Reduction of Second Order Piezoelectric Energy Harvester Model
}

\author{
Chengdong Yuan ${ }^{*, * *}$ Siyang $\mathbf{H u}^{*, * *}$ \\ Alessandro Castagnotto ${ }^{* * *}$ Boris Lohmann ${ }^{* * *}$ \\ Tamara Bechtold ${ }^{* * *}$ \\ * Department of Engineering, Jade University of Applied Sciences, \\ Friedrich-Paffrath-Str. 101, D-26389 Wilhelmshaven. \\ (e-mail: \{chengdong.yuan, siyang.hu, tamara.bechtold\}@jade-hs.de) \\ ** Institute for Electronic Appliances and Circuits, University of \\ Rostock, Albert-Einstein-Str. 2, 18059 Rostock. \\ *** Chair of Automatic Control, Technical University of Munich, \\ Boltzmannstr. 15, D-85748 Garching. \\ (e-mail: $\{$ a.castagnotto, lohmann\}@tum.de)
}

Keywords: Multi-physical system, Model reduction, Krylov subspace, Schur complement.

\section{INTRODUCTION}

As energy efficiency becomes increasingly important in everyday life, different energy harvesting systems are being developed to recycle the energy wasted in the surrounding. By doing so, these devices act as independent power supplies for wireless micro-devices, as an alternative to batteries. In (Wang et al., 2012), the authors have introduced an energy harvester, which transforms vibrations into electrical energy, using the piezoelectric effect.

Kudryavtsev et al. (2015) showed that direct application of one-sided Krylov-subspace-based model order reduction (MOR) (Bai and Su, 2005; Salimbahrami and Lohmann, 2006; Gugercin et al., 2013) to the harvester model may lead to unstable reduced models. Therefore, the authors suggested a new approach called 'MOR after Schur' as they were able to obtain stable reduced models, when a Schur complement transformation was performed before MOR. However, they also stated that a Schur complement transformation increases the number of non-zero entries in the stiffness matrix and therefore the computational effort. To tackle this issue Benner et al. (2016) suggest to undo the Schur complement during the computation of the projection matrices to retake advantage of the sparse structure.

In this work, we consider an alternative approach for efficient one-sided reduction of the second-order piezoelectric energy harvester model, based on the work on first order systems in (Castagnotto et al., 2015), and establish an implicit Schur complement transformation.

\section{MOR AFTER SCHUR}

The system-level representation of the energy harvester model is shown in Fig. 1. It contains one mechanical input named displ, three mechanical outputs named centre, south, north and two electrical ports named el1, el2.

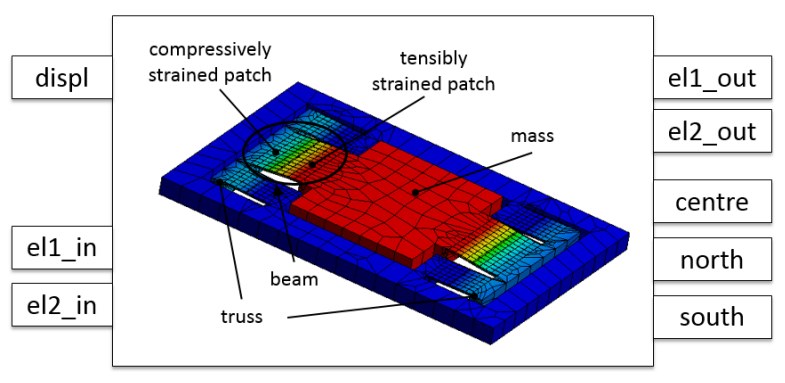

Fig. 1. System-level representation of piezoelectric energy harvester similar to (Kudryavtsev et al., 2015).

After the spatial discretization of governing partial differential equations, the piezoelectric model can be described as follows (Kudryavtsev et al., 2015):

$$
\left\{\begin{array}{l}
\underbrace{\left[\begin{array}{cc}
M_{11} & 0 \\
0 & 0
\end{array}\right]}_{M}\left[\begin{array}{c}
\ddot{x}_{1} \\
\ddot{x}_{2}
\end{array}\right]+\underbrace{\left[\begin{array}{cc}
E_{11} & 0 \\
0 & 0
\end{array}\right]}_{E}\left[\begin{array}{l}
\dot{x}_{1} \\
\dot{x}_{2}
\end{array}\right]+\underbrace{\left[\begin{array}{ll}
K_{11} & K_{12} \\
K_{21} & K_{22}
\end{array}\right]}_{K}\left[\begin{array}{l}
x_{1} \\
x_{2}
\end{array}\right]=\underbrace{\left[\begin{array}{l}
B_{1} \\
B_{2}
\end{array}\right]}_{B} u \\
y=\underbrace{\left[\begin{array}{ll}
C_{1} & C_{2}
\end{array}\right]}_{C}\left[\begin{array}{l}
x_{1} \\
x_{2}
\end{array}\right]
\end{array}\right.
$$

$M, E \in \mathbb{R}^{(n+k) \times(n+k)}$ are the structural mass and damping matrix. $K_{11} \in \mathbb{R}^{n \times n}$ is the structural stiffness matrix, $K_{12} \in \mathbb{R}^{n \times k}, K_{21} \in \mathbb{R}^{k \times n}$ are the piezoelectric coupling matrices and $K_{22} \in \mathbb{R}^{k \times k}$ is the dielectric conductivity matrix. $x_{1} \in \mathbb{C}^{n}$ and $x_{2} \in \mathbb{C}^{k}$ are parts of the state vector representing nodal displacement and electrical potentials. $u \in \mathbb{R}^{l}$ is the vector of input load, $B_{1} \in \mathbb{R}^{n \times l}$ and $B_{2} \in \mathbb{R}^{k \times l}$ are parts of input matrix $B . y \in \mathbb{C}^{m}$ is the user defined output vector, $C_{1} \in \mathbb{R}^{m \times n}$ and $C_{2} \in \mathbb{R}^{m \times k}$ are parts of gathering matrix $C$.

When a Schur complement transformation is applied before the MOR process, the electrical domain related state vector $x_{2}$ can be eliminated by $x_{2}=K_{22}^{-1}\left(B_{2} u-K_{21} x_{1}\right)$, where given $K_{22}$ is invertible, and the system is then transformed into: 


$$
\left\{\begin{array}{l}
M_{11} \ddot{x}_{1}+E_{11} \dot{x}_{1}+\underbrace{\left(K_{11}-K_{12} K_{22}^{-1} K_{21}\right)}_{=: K_{s}} x_{1}=\underbrace{\left(B_{1}-K_{12} K_{22}^{-1} B_{2}\right)}_{=: B_{s}} u \\
y=\underbrace{\left(C_{1}-C_{2} K_{22}^{-1} K_{21}\right)}_{=: C_{s}} x_{1}+\underbrace{\left(C_{2} K_{22}^{-1} B_{2}\right)}_{=: D_{s}} u
\end{array}\right.
$$

For one-side reduction $(W=V)$, the projection matrix $V$ can be obtained from moment matching at the expansion point $\omega=0$ (Salimbahrami and Lohmann, 2006). The reduced model resulting from the projection can then be written as:

$$
\left\{\begin{array}{l}
V^{T} M_{11} V \ddot{z}+V^{T} E_{11} V \dot{z}+V^{T} K_{s} V z=V^{T} B_{s} u \\
y=C_{s} V z+D_{s} u
\end{array}\right.
$$

\section{MOR AFTER IMPLICIT SCHUR}

In (Castagnotto et al., 2015), the authors show that an equivalent system for the first-order semi-explicit system can be established by projecting the input matrix onto the right deflating subspace corresponding to the finite eigenvalues. Using this new formulation, the Schur complement is implicitly performed during projection, as stated in the following result.

Theorem 1. The reduced model (3) can be obtained by reducing the equivalent system (4):

$$
\left\{\begin{array}{l}
\underbrace{\left[\begin{array}{cc}
M_{11} & 0 \\
0 & 0
\end{array}\right]}_{M}\left[\begin{array}{c}
\ddot{x}_{1} \\
\ddot{x}_{2}
\end{array}\right]+\underbrace{\left[\begin{array}{cc}
E_{11} & 0 \\
0 & 0
\end{array}\right]}_{E}\left[\begin{array}{c}
\dot{x}_{1} \\
\dot{x}_{2}
\end{array}\right]+\underbrace{\left[\begin{array}{cc}
K_{11} & K_{12} \\
K_{21} & K_{22}
\end{array}\right]}_{K}\left[\begin{array}{c}
x_{1} \\
x_{2}
\end{array}\right]=\underbrace{\left[\begin{array}{c}
B_{s} \\
0
\end{array}\right]}_{\widetilde{B}} u \\
y=\underbrace{\left[\begin{array}{ll}
C_{1} & C_{2}
\end{array}\right]}_{C}\left[\begin{array}{l}
x_{1} \\
x_{2}
\end{array}\right]+D_{s} u
\end{array}\right.
$$

where the Schur complement transformation is only performed on the input matrix $\widetilde{B}$ and the feed-through matrix $D_{s}$ is added.

The equality of the reduced models to (3) can then easily be shown by straightforward projection of (4) with its oneside Krylov subspace projection matrix. Note that (4) and (1) share the same transfer function, hence preserving the moment matching property.

\section{NUMERICAL RESULTS AND CONCLUSION}

Fig. 2 illustrates the full model's frequency response of the electrical outputs to the mechanical displacement excitation, which is visibly well matched by the frequency responses of the reduced models (reduce order $r=30$ ) from both MOR after normal and implicit Schur. Both reduced models are obtained using first order one-sided Arnoldi at the expansion point $\omega=0$ and with proportional damping (Rudnyi et al., 2004). Furthermore, Table 1 shows that the computational time of the MOR process can be significantly reduced (six times quicker) using implicit Schur, since the structure of the stiffness Matrix is preserved

\section{REFERENCES}

Bai, Z. and Su, Y. (2005). Dimension reduction of largescale second-order dynamical systems via a secondorder Arnoldi method. SIAM Journal on Scientific Computing, 26(5), 1692-1709.
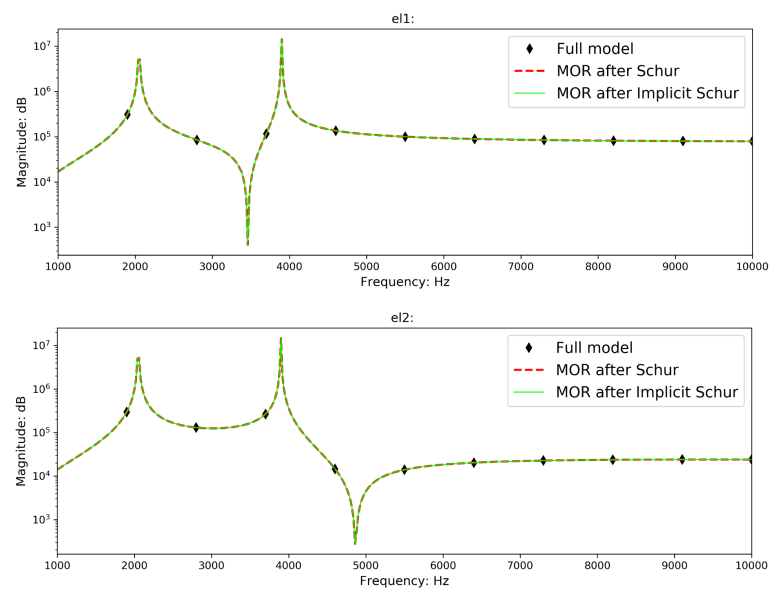

Fig. 2. Frequency response of the electrical outputs el1_out and el2_out with displacement excitation displ of full and reduced models.

Table 1. Computational time of MOR for normal and implicit Schur complement transformed system.

\begin{tabular}{ccc}
\hline & $\begin{array}{c}\text { MOR after } \\
\text { Schur }\end{array}$ & $\begin{array}{c}\text { MOR after } \\
\text { Implicit Schur }\end{array}$ \\
\hline $\begin{array}{c}\text { Computational } \\
\text { Time (s) }\end{array}$ & 213.241 & 33.827 \\
\hline
\end{tabular}

Benner, P., Saak, J., and Uddin, M.M. (2016). Reducedorder modeling of index-1 vibrational systems using interpolatory projections. In Computer and Information Technology (ICCIT), 2016 19th International Conference on, 134-138. IEEE.

Castagnotto, A., Panzer, H.K., Reinsch, K.D., and Lohmann, B. (2015). Stability-preserving, adaptive model order reduction of daes by Krylov-subspace methods. arXiv preprint arXiv:1508.07227.

Gugercin, S., Stykel, T., and Wyatt, S. (2013). Model reduction of descriptor systems by interpolatory projection methods. SIAM Journal on Scientific Computing, 35(5), B1010-B1033.

Kudryavtsev, M., Rudnyi, E.B., Korvink, J.G., Hohlfeld, D., and Bechtold, T. (2015). Computationally efficient and stable order reduction methods for a large-scale model of mems piezoelectric energy harvester. Microelectronics Reliability, 55(5), 747-757.

Rudnyi, E.B., Lienemann, J., Greiner, A., and Korvink, J.G. (2004). mor4ansys: Generating compact models directly from ansys models. In Technical Proceedings of the 2004 Nanotechnology Conference and Trade Show, Nanotech, volume 2, 279-282.

Salimbahrami, B. and Lohmann, B. (2006). Order reduction of large scale second-order systems using Krylov subspace methods. Linear Algebra and its Applications, 415(2-3), 385-405.

Wang, Z., Matova, S., Elfrink, R., Jambunathan, M., De Nooijer, C., van Schaijk, R., and Vullers, R. (2012). A piezoelectric vibration harvester based on clampedguided beams. In Micro Electro Mechanical Systems (MEMS), 2012 IEEE 25th International Conference on, 1201-1204. IEEE. 